\title{
Metric fluctuations and their evolution during inflation
}

\author{
M. Anabitarte ${ }^{1, \mathrm{a}}$, M. Bellini ${ }^{1,2, \mathrm{~b}}$ \\ 1 Departamento de Física, Facultad de Ciencias Exactas y Naturales Universidad Nacional de Mar del Plata, Funes 3350, \\ (7600) Mar del Plata, Buenos Aires, Argentina \\ 2 Consejo Nacional de Investigaciones Científicas y Técnicas (CONICET)
}

Received: 10 December 2003 /

Published online: 23 March 2004 - (C) Springer-Verlag / Società Italiana di Fisica 2004

\begin{abstract}
We discuss the evolution of the fluctuations in a symmetric $\phi_{\mathrm{c}}$-exponential potential which provides a power-law expansion during inflation using both the gauge-invariant field $\Phi$ and the SasakiMukhanov field.
\end{abstract}

\section{Introduction and motivation}

It is now widely accepted that the dominant cause of structure in the Universe is a spatial perturbation. This perturbation is present on cosmological scales a few Hubble times before these scales enter the horizon, at which stage it is time-independent with an almost flat spectrum. One of the main objectives of theoretical cosmology is to understand its origin [1]. The usual assumption is that the curvature perturbation originates during inflation of the slow-rolling inflaton field. As cosmological scales leave the horizon, the quantum fluctuation is converted to a classical gaussian perturbation with an almost flat spectrum, generating immediately the required curvature perturbation which is constant until the approach of horizon entry [2]. This idea has the advantage that the prediction for the spectrum is independent of what goes on between the end of inflation and horizon entry. The spectrum depends only on the form of the potential and on the theory of gravity during inflation, providing, therefore, a direct probe of conditions during this era.

Stochastic inflation has played an important role in inflationary cosmology in the last two decades. It proposes to describe the dynamics of this quantum field on the basis of two pieces: the homogeneous and inhomogeneous components [3-10]. Usually the homogeneous one is interpreted as a classical field $\phi_{\mathrm{c}}(t)$ that arises from the vacuum expectation value of the quantum field. The inhomogeneous components $\phi(\mathbf{x}, t)$ are the quantum fluctuations. The field that takes into account only the modes with wavelengths larger than the now observable universe is called a coarse-grained field and its dynamics is described by a second-order stochastic equation $[10,11]$. Since these perturbations are classical on super Hubble scales, in this sector one can make a standard stochastic treatment for

\footnotetext{
a e-mail: anabitar@mdp.edu.ar

b e-mail: mbellini@mdp.edu.ar
}

the coarse-grained matter field [10]. The IR sector is very important, because the spatially inhomogeneities in super Hubble inflationary scales would explain the present day observed matter structure in the universe.

In this work we consider gauge-invariant fluctuations of the metric in the early inflationary universe [2]. Metric fluctuations are here considered in the framework of linear perturbative corrections. The scalar metric perturbations are spin-zero projections of the graviton, which only exists in non-vacuum cosmologies. The issue of gauge invariance becomes critical when we attempt to analyze how the scalar metric perturbations produced in the early universe influence a background globally flat isotropic and homogeneous universe. This allows us to formulate the problem of the amplitude for the scalar metric perturbations on the evolution of the background Friedmann-Robertson-Walker (FRW) universe in a coordinate-independent manner at every moment in time. On the other hand, the Sasaki-Mukhanov (SM) field takes into account both: metric and inflaton fluctuations [12]. One of the aims of this work is the study of the evolution of the SM field during inflation to make a comparison with gauge-invariant metric fluctuations.

\section{Fluctuations}

Matter field fluctuations are responsible for metric fluctuations around the background FRW metric. When these metric fluctuations do not depend on the gauge, the perturbed globally flat isotropic and homogeneous universe is described by [2]

$$
\mathrm{d} s^{2}=(1+2 \psi) \mathrm{d} t^{2}-a^{2}(t)(1-2 \Phi) \mathrm{d} x^{2},
$$

where $a$ is the scale factor of the universe and $(\psi, \Phi)$ are the gauge-invariant perturbations of the metric. In the particular case where the tensor $T_{\alpha \beta}$ is diagonal, one obtains $\Phi=\psi[2]$. We consider a semiclassical expansion for the 
inflaton field $\varphi(\mathbf{x}, t)=\phi_{\mathrm{c}}(t)+\phi(\mathbf{x}, t)[10]$, with expectation values $\langle 0|\varphi| 0\rangle=\phi_{\mathrm{c}}(t)$ and $\langle 0|\phi| 0\rangle=0$. Here, $|0\rangle$ is the vacuum state. Due to $\langle 0|\Phi| 0\rangle=0$, the expectation value of the metric (1) gives the background metric that describes a flat FRW spacetime: $\left\langle\mathrm{d} s^{2}\right\rangle=\mathrm{d} t^{2}-a^{2} \mathrm{~d} x^{2}$.

After linearizing the Einstein equations in terms of $\phi$ and $\Phi$, one obtains

$$
\begin{gathered}
\ddot{\Phi}+\left(H-2 \frac{\ddot{\phi}_{\mathrm{c}}}{\dot{\phi}_{\mathrm{c}}}\right) \dot{\Phi}-\frac{1}{a^{2}} \nabla^{2} \Phi \\
+2\left(\dot{H}-H \frac{\ddot{\phi}_{\mathrm{c}}}{\dot{\phi}_{\mathrm{c}}}\right) \Phi=0, \\
\frac{1}{a} \frac{\mathrm{d}}{\mathrm{d} t}(a \Phi)_{, \beta}=\frac{4 \pi}{M_{\mathrm{P}}^{2}}\left(\dot{\phi}_{\mathrm{c}} \phi\right)_{, \beta}, \\
\ddot{\phi}+3 H \dot{\phi}-\frac{1}{a^{2}} \nabla^{2} \phi+V^{\prime \prime}\left(\phi_{\mathrm{c}}\right) \phi \\
+2 V^{\prime}\left(\phi_{\mathrm{c}}\right) \Phi-4 \dot{\phi}_{\mathrm{c}} \dot{\Phi}=0,
\end{gathered}
$$

where $\beta=0,1,2,3, a$ is the scale factor of the universe and the prime denotes the derivative with respect to $\phi_{\mathrm{c}}$. The dynamics of $\phi_{\mathrm{c}}$ is given by the equations

$$
\ddot{\phi}_{\mathrm{c}}+3 H \dot{\phi}_{\mathrm{c}}+V^{\prime}\left(\phi_{\mathrm{c}}\right)=0, \quad \dot{\phi}_{\mathrm{c}}=-\frac{M_{\mathrm{P}}^{2}}{4 \pi} H^{\prime},
$$

and $H=\dot{a} / a$ is the Hubble parameter. Furthermore, the scalar potential can be written in terms of the Hubble parameter:

$$
V\left(\phi_{\mathrm{c}}\right)=\frac{3 M_{\mathrm{P}}^{2}}{8 \pi}\left[H^{2}-\frac{M_{\mathrm{P}}^{2}}{12 \pi}\left(H^{\prime}\right)^{2}\right] .
$$

Equation (2) can be simplified by introducing the field $Q=\mathrm{e}^{1 / 2 \int\left[H-2 \ddot{\phi}_{\mathrm{c}} / \phi_{\mathrm{c}}\right] \mathrm{d} t} \Phi$,

$$
\begin{aligned}
\ddot{Q}- & \frac{1}{a^{2}} \nabla^{2} Q-\left[\frac{1}{4}\left(H-2 \frac{\ddot{\phi}_{\mathrm{c}}}{\dot{\phi}_{\mathrm{c}}}\right)^{2}\right. \\
& \left.+\frac{1}{2}\left[\dot{H}-2 \frac{\mathrm{d}}{\mathrm{d} t}\left(\frac{\ddot{\phi}_{\mathrm{c}}}{\dot{\phi}_{\mathrm{c}}}\right)\right]-2\left(\dot{H}-H \frac{\ddot{\phi}_{\mathrm{c}}}{\dot{\phi}_{\mathrm{c}}}\right)\right] Q=0 .
\end{aligned}
$$

This field can be expanded in terms of the modes $Q_{k}=$ $\mathrm{e}^{\mathrm{i} \mathbf{k} \cdot \mathbf{x}} \xi_{k}(t)$ :

$$
Q(\mathbf{x}, t)=\frac{1}{(2 \pi)^{3 / 2}} \int \mathrm{d}^{3} k\left[\alpha_{k} Q_{k}(\mathbf{x}, t)+\alpha_{k}^{\dagger} Q_{k}^{*}(\mathbf{x}, t)\right],
$$

where $\alpha_{k}$ and $\alpha_{k}^{\dagger}$ are the annihilation and creation operators that comply with the commutation relations

$$
\begin{aligned}
{\left[\alpha_{k}, \alpha_{k^{\prime}}^{\dagger}\right] } & =\delta^{(3)}\left(k-k^{\prime}\right), \\
{\left[\alpha_{k}, \alpha_{k^{\prime}}\right] } & =\left[\alpha_{k}^{\dagger}, \alpha_{k^{\prime}}^{\dagger}\right]=0 .
\end{aligned}
$$

The equation for the modes $Q_{k}$ is

$$
\ddot{Q}_{k}+\omega_{k}^{2}(t) Q_{k}=0,
$$

where $\omega_{k}^{2}=a^{-2}\left(k^{2}-k_{0}^{2}\right)$ is the squared time-dependent frequency and $k_{0}$ separates the infrared and ultraviolet sectors and is given by

$$
\begin{gathered}
\frac{k_{0}^{2}}{a^{2}}=\frac{1}{4}\left(H-2 \frac{\ddot{\phi}_{\mathrm{c}}}{\dot{\phi}_{\mathrm{c}}}\right)^{2}+\frac{1}{2}\left[\dot{H}-2 \frac{\mathrm{d}}{\mathrm{d} t}\left(\frac{\ddot{\phi}_{\mathrm{c}}}{\dot{\phi}_{\mathrm{c}}}\right)\right] \\
-2\left(\dot{H}-H \frac{\ddot{\phi}_{\mathrm{c}}}{\dot{\phi}_{\mathrm{c}}}\right) .
\end{gathered}
$$

Since the field $Q$ satisfies a Klein-Gordon-like equation on a FRW background metric, $\left\langle\mathrm{d} s^{2}\right\rangle=\mathrm{d} t^{2}-a^{2} \mathrm{~d} x^{2}$, it also satisfies the commutation relation

$$
\left[Q(\mathbf{x}, t) \dot{Q}\left(\mathbf{x}^{\prime}, t\right)\right]=\mathrm{i} \delta^{(3)}\left(\mathbf{x}-\mathbf{x}^{\prime}\right) .
$$

This implies that the modes $Q_{k}$ are renormalized by the expression

$$
\dot{Q}_{k}^{*} Q_{k}-\dot{Q}_{k} Q_{k}^{*}=\mathrm{i}
$$

\subsection{Particular solutions}

If the inflaton field oscillates around the minimum of the potential at the end of inflation the particular solutions when $\dot{\phi}_{\mathrm{c}}=0$ and $\ddot{\phi}_{\mathrm{c}}=0$ are very important.

On the points $\dot{\phi}_{\mathrm{c}}=0$ we obtain $Q_{k}=0$. However, the solutions for $\Phi_{k}$ are non-zero:

$$
\Phi_{k}=a^{-1} \phi_{k}^{0},
$$

where $\phi_{k}^{0}$ is the initial amplitude for $\Phi_{k}$, for each wavenumber $k$. This means that the amplitude of each mode decreases exponentially with time.

Another interesting particular solution is located at the points $\ddot{\phi}_{\mathrm{c}}=0$, when the field is at the minumum of the potential. In these points (11) adopts the form

$$
\ddot{Q}_{k}+\left[\frac{k^{2}}{a^{2}}-\left(\frac{H^{2}}{4}-\frac{3}{2} \dot{H}\right)\right] Q_{k}=0,
$$

where $\Phi_{k}=a^{-1 / 2} Q_{k}$.

\subsection{The Sasaki-Mukhanov field}

A manner to study both, metric and inflaton fluctuations, can be made by means of the SM field [12]: $S=\phi+\frac{\dot{\phi}_{\mathrm{c}}}{H_{\mathrm{c}}} \Phi$. The modes of this field obey the following equation:

$\ddot{S}_{k}(t)+3 H \dot{S}_{k}(t)+\left[\frac{k^{2}}{a^{2}}+V^{\prime \prime}+2 \frac{\mathrm{d}}{\mathrm{d} t}\left(\frac{\dot{H}}{H}+3 H\right)\right] S_{k}(t)=0$,

where the modes $S_{k}$ comply with the renormalization condition

$$
\dot{S}_{k}^{*} S_{k}-\dot{S}_{k} S_{k}^{*}=\frac{\mathrm{i}}{a^{3}},
$$

so that $\left[S(\mathbf{x}, t), \dot{S}\left(\mathbf{x}^{\prime}, t\right)\right]=\frac{\mathrm{i}}{a^{3}} \delta^{(3)}\left(\mathbf{x}-\mathbf{x}^{\prime}\right)$. 


\subsection{Power spectrum}

One can estimate the power spectrum of the fluctuations for the fields $\Phi$ and $S$. The spectrum of the fluctuations for $\Phi$ is

$$
\mathcal{P}_{\Phi}=\frac{k^{3}}{3 \pi^{2}}\left|\Phi_{k}(t)\right|^{2},
$$

whilst the power spectrum for the SM field is the same as that of the inflation field:

$$
\mathcal{P}_{S}=\frac{k^{3}}{2 \pi^{2}}\left|S_{k}(t)\right|^{2} .
$$

It is well known from the experimental data [13] that the universe has a scale invariant power spectrum on cosmological scales.

\section{An example.}

\section{Symmetric exponential $\phi_{\mathrm{c}}$-potential: power-law inflation}

As a first example we consider a scalar potential given by $V\left(\phi_{\mathrm{c}}\right)=V_{0} \mathrm{e}^{2 \alpha\left|\phi_{\mathrm{c}}\right|}$, where $\alpha^{2}=\frac{4 \pi}{M_{\mathrm{P}}^{2} p}$ gives the relation between $\alpha$ and the power of the expansion $p$. This potential is related to a scale factor that evolves as $a \sim t^{p}$ (with constant power $p$ ), which corresponds to a Hubble parameter $H(t)=p / t$, which can be written in terms of the scalar field

$$
H_{\mathrm{c}}=\frac{\pi}{M_{\mathrm{P}}}\left(\frac{32 V_{0}}{12 \pi-\alpha^{2} M_{\mathrm{P}}^{2}}\right)^{1 / 2} \mathrm{e}^{\alpha\left|\phi_{\mathrm{c}}\right|},
$$

where $V_{0}=\frac{3 M_{\mathrm{P}}^{2}}{8 \pi} H_{\mathrm{e}}^{2}\left[\frac{12 \pi-M_{\mathrm{P}}^{2} \alpha^{2}}{12 \pi}\right]$ and $H_{\mathrm{e}}=p / t_{\mathrm{e}}$ is the value of the Hubble parameter at the end of inflation. The temporal evolution for $\left|\phi_{\mathrm{c}}(t)\right|$ is given by

$$
\left|\phi_{\mathrm{c}}(t)\right|=\left|\phi_{0}\right|-\frac{1}{\alpha} \ln \left(\frac{t}{t_{0}}\right)
$$

where $t \geq t_{0}$. Since $\dot{\phi}_{\mathrm{c}}=-\operatorname{sgn}\left(\phi_{\mathrm{c}}\right) \frac{1}{\alpha t}$ and $\ddot{\phi}_{\mathrm{c}}=\operatorname{sgn}\left(\phi_{\mathrm{c}}\right) \frac{1}{\alpha t^{2}}$ (we assume $\operatorname{sgn}\left(\phi_{\mathrm{c}}\right)= \pm 1$ for $\phi_{\mathrm{c}}$ positive and negative, respectively), the equation that describes the evolution for $\Phi$ results:

$$
\ddot{\Phi}+\frac{(p+2)}{t} \dot{\Phi}-\frac{1}{a^{2}} \nabla^{2} \Phi=0 .
$$

After we make the transformation $Q=\Phi \mathrm{e}^{\int(p+2) t^{-1} \mathrm{~d} t}$, we obtain the differential equation for $Q$ :

$$
\ddot{Q}-\frac{1}{a^{2}} \nabla^{2} Q-\left[\frac{p}{2}\left(\frac{p}{2}+1\right) t^{-2}\right] Q=0 .
$$

The general solution for the modes $Q_{k}(t)$ is

$$
Q_{k}(t)=C_{1} \sqrt{\frac{t}{t_{0}}} \mathcal{H}_{\nu_{1}}^{(1)}[x(t)]+C_{2} \sqrt{\frac{t}{t_{0}}} \mathcal{H}_{\nu_{1}}^{(2)}[x(t)],
$$

where $\left(C_{1}, C_{2}\right)$ are constants, $\left(\mathcal{H}_{\nu_{1}}^{(1)}[x], H_{\nu_{1}}^{(2)}[x]\right)$ are the Hankel functions of the first and second kind, with $x(t)=$ $\frac{t_{o}^{p} k}{a_{0}(p-1) t^{p-1}}$ and $\nu_{1}=\frac{p+1}{2(p-1)}$. Using the renormalization condition $\dot{Q}_{k}^{*} Q_{k}-\dot{Q}_{k} Q_{k}^{*}=\mathrm{i}$, we obtain the Bunch-Davis vacuum [14] solution $\left(C_{1}=0, C_{2}=\sqrt{\frac{\pi}{2(p-1)}}\right)$,

$$
Q_{k}(t)=\sqrt{\frac{\pi}{2}} \sqrt{\frac{t}{t_{0}(p-1)}} \mathcal{H}_{\nu_{1}}^{(2)}[x(t)] .
$$

In the UV sector the function $\mathcal{H}_{\nu_{1}}^{(2)}[x]$ adopts the asymptotic expression (i.e., for $x \gg 1$ )

$$
\begin{aligned}
\mathcal{H}_{\nu_{1}}^{(2)}[x] \simeq \sqrt{\frac{2}{\pi x}}\left[\cos \left(x-\nu_{1} \pi / 2-\pi / 4\right)\right. \\
\left.-\mathrm{i} \sin \left(x-\nu_{1} \pi / 2-\pi / 4\right)\right],
\end{aligned}
$$

whilst on the IR sector (i.e., for $x \ll 1$ ) it tends asymptotically to

$$
\mathcal{H}_{\nu_{1}}^{(2)}[x] \simeq \frac{1}{\Gamma\left(\nu_{1}+1\right)}\left(\frac{x}{2}\right)^{\nu_{1}}-\frac{\mathrm{i}}{\pi} \Gamma\left(\nu_{1}\right)\left(\frac{x}{2}\right)^{-\nu_{1}} .
$$

The $\Phi$-squared field fluctuations on the IR sector are $\left(\left\langle\Phi^{2}\right\rangle\right)_{\mathrm{IR}}=\frac{1}{2 \pi^{2}} \int_{0}^{\epsilon k_{0}(t)} \mathrm{d} k k^{2}\left|\Phi_{k}\right|^{2}$, and become

$\left(\left\langle\Phi^{2}\right\rangle\right)_{\mathrm{IR}}$

$\simeq \frac{1}{4}\left\{\frac{\left[\frac{t_{0}^{p}}{2 a_{0}(p-1)}\right]^{2 \nu_{1}}\left[\frac{\epsilon a_{0} \sqrt{\frac{p}{2}\left(\frac{p}{2}+1\right)}}{t_{0}^{p}}\right]^{\frac{2(2 p-1)}{p-1}}}{\pi t_{0} \Gamma^{2}\left(\frac{3 p-1}{2(p-1)}\right)(3 p-1)}\right.$

$$
\left.+\frac{\Gamma^{2}\left(\nu_{1}\right)\left[\frac{t_{0}^{p}}{2 a_{0}(p-1)}\right]^{-2 \nu_{1}}\left[\frac{\epsilon a_{0} \sqrt{\frac{p}{2}\left(\frac{p}{2}+1\right)}}{t_{0}^{p}}\right]^{\frac{2(p-2)}{p-1}}}{\pi^{3} t_{0}(p-3)}\right\} t^{3-2 \nu_{1}},
$$

where $\epsilon=k_{\max }^{(\mathrm{IR})} / k_{\mathrm{P}} \ll 1$ is a dimensionless constant, $k_{\max }^{(\mathrm{IR})}=k_{0}\left(t_{*}\right)$ at the moment $t_{*}$ of the horizon entry, and $k_{\mathrm{P}}$ is the Planckian wavenumber (i.e., the scale we choose as a cut-off of the whole spectrum). The power spectrum on the IR sector is $\left.\mathcal{P}_{\Phi}\right|_{\mathrm{IR}} \sim k^{3-2 \nu_{1}}$. Note that $\left(\left\langle\Phi^{2}\right\rangle\right)_{\mathrm{IR}}$ increases for $p>2$, so that to have the IR squared $\Phi$-fluctuations remain almost constant on cosmological scales we need $p \simeq 2$. We find that a power close to $p=2$ gives us a scale invariant power spectrum (i.e., with $\nu_{1} \simeq 3 / 2$ for $\left(\left\langle\Phi^{2}\right\rangle\right)_{\text {IR }}$. Furthermore, density fluctuations for the matter energy density are given by $\delta \rho / \rho=-2 \Phi$, so that $\left\langle\delta \rho^{2}\right\rangle^{1 / 2} /\langle\rho\rangle \sim\left\langle\Phi^{2}\right\rangle^{1 / 2}$.

On the other hand, in the UV sector these fluctuations are given by

$$
\left(\left\langle\Phi^{2}\right\rangle\right)_{\mathrm{UV}} \simeq \frac{a_{0}}{4 t_{0}^{p+1} \pi^{2}}\left\{\frac{k_{\mathrm{P}}^{2}}{t^{2}}-\frac{a_{0}^{2}}{t^{2 p}}\left[\frac{p}{2}\left(\frac{p}{2}+1\right)\right]\right\} t^{3-2 \nu_{1}} .
$$


The power spectrum in this sector goes as $\left.\mathcal{P}_{\Phi}\right|_{\mathrm{UV}} \sim k^{4}$. We observe from (30) that $\left(\left\langle\Phi^{2}\right\rangle\right)_{\mathrm{UV}}$ increases during inflation for $p>3$. From the results (29) and (30) we obtain $1<p \leq 2$, because a power-law $p>2$ could give a very inhomogeneous universe on cosmological scales. Since $\left(\left\langle\Phi^{2}\right\rangle\right)_{\mathrm{UV}} \geq 0$, we obtain the condition

$$
k_{\mathrm{P}}^{2}-\frac{a_{0}^{2}}{t^{2(p-1)}}\left[\frac{p}{2}\left(\frac{p}{2}+1\right)\right] \geq 0 .
$$

If $a_{0}=H_{0}^{-1}$ ( $H_{0}$ is the initial value of the Hubble parameter), inflation should end at $t=t_{\mathrm{e}}$, where

$$
t_{\mathrm{e}} \simeq\left[\frac{k_{\mathrm{P}} H_{0}}{\sqrt{\frac{p}{2}\left(\frac{p}{2}+1\right)}}\right]^{\frac{1}{p-1}} .
$$

For example, for $k_{\mathrm{P}} H_{0}=10^{11} M_{\mathrm{P}}$ and $p=2$, we obtain $t_{\mathrm{e}} \simeq 5.810^{10} M_{\mathrm{P}}^{-1}$.

Now we can study the evolution of the SM field fluctuations $\left\langle S^{2}\right\rangle$. Equation (17) written explicitly for the model we are studying is

$$
\ddot{S}_{k}+3 p t^{-1} \dot{S}_{k}+\left(k H_{0}\right)^{2}\left(\frac{t_{0}}{t}\right)^{2 p} S_{k}=0 .
$$

Note that the last term inside the brackets in (17) becomes null. The general solution of (32) can be written in terms of the Hankel functions

$$
\begin{aligned}
S_{k}(t)= & A\left(\frac{t}{t_{0}}\right)^{\left.\frac{1}{2}(1-3 p)\right)} \mathcal{H}_{\nu_{2}}^{(1)}\left[\frac{k H_{0} t_{0}^{p} t^{1-p}}{p-1}\right] \\
& +B\left(\frac{t}{t_{0}}\right)^{\left.\frac{1}{2}(1-3 p)\right)} \mathcal{H}_{\nu_{2}}^{(2)}\left[\frac{k H_{0} t_{0}^{p} t^{1-p}}{p-1}\right],
\end{aligned}
$$

where $\nu_{2}=\frac{(3 p-1)}{2(p-1)}$. If we adopt the Buch-Davis vacuum [14]: $A=0, B=\sqrt{\frac{\pi}{2(p-1)}}$, we obtain

$$
S_{k}(t)=\sqrt{\frac{\pi t^{1-3 p}}{2(p-1) t_{0}^{1-3 p}}} \mathcal{H}_{\nu_{2}}^{(2)}\left[\frac{k H_{\mathrm{e}} t_{0}^{p} t^{1-p}}{p-1}\right] .
$$

The power spectra in the extreme sectors of the spectrum go as

$$
\begin{aligned}
& \left.\mathcal{P}_{S}\right|_{\mathrm{UV}} \sim k^{4}, \\
& \left.\mathcal{P}_{S}\right|_{\mathrm{IR}} \sim k^{3-2 \nu_{2}} .
\end{aligned}
$$

Note that $\left.\mathcal{P}_{S}\right|_{\text {IR }}$ is scale invariant for $\nu_{2}=3 / 2$, which corresponds with $p \rightarrow \infty$. The squared $S$-fluctuations on both the UV and IR sectors are

$$
\begin{aligned}
\left(\left\langle S^{2}\right\rangle\right)_{\mathrm{UV}} \simeq & \frac{t^{-2} t_{0}}{4 \pi^{2} H_{\mathrm{e}}^{3}} \\
& \times\left[k_{\mathrm{P}}^{2} H_{\mathrm{e}}^{2}\left(\frac{t_{0}}{t}\right)^{2 p} t^{2}-\left(\frac{9}{4} p^{2}-\frac{15}{2} p+2\right)\right]
\end{aligned}
$$

$$
\left(\left\langle S^{2}\right\rangle\right)_{\mathrm{IR}} \simeq \frac{\Gamma^{2}\left(\nu_{2}\right) H_{\mathrm{e}}^{-3} \epsilon^{3-2 \nu_{2}}\left(\frac{9}{4} p^{2}-\frac{15}{2} p+2\right)^{\frac{3-2 \nu_{2}}{2}}}{8 \pi^{3}(p-1)^{1-2 \nu_{2}}\left(3-2 \nu_{2}\right) t_{0}} t^{-2} .
$$

Since $\left(\left\langle S^{2}\right\rangle\right)_{\mathrm{UV}} \geq 0$ during inflation, from (38) we obtain the condition

$$
\left[k_{\mathrm{P}}^{2} H_{\mathrm{e}}^{2}\left(\frac{t_{0}}{t}\right)^{2 p} t^{2}-\left(\frac{9}{4} p^{2}-\frac{15}{2} p+2\right)\right] \geq 0 .
$$

From this condition we obtain the time at which inflation ends. Hence, one obtains

$$
t_{\mathrm{e}} \simeq\left(\frac{k_{\mathrm{P}} H_{\mathrm{e}}}{\sqrt{\frac{9}{4} p^{2}-\frac{15}{2} p+2}}\right)^{\frac{1}{p-1}}
$$

where, since we require $\frac{9}{4} p^{2}-\frac{15}{2} p+2 \geq 0, p \geq 3.04$ must hold. For example, for $p=4$ and $k_{\mathrm{P}} H_{\mathrm{e}}=10^{11} M_{\mathrm{P}}$, one obtains $t_{\mathrm{e}} \simeq 2.810^{3} M_{\mathrm{P}}^{-1}$, which is incompatible with the value obtained from the evolution for $\Phi$. On the other hand $\left(\left\langle S^{2}\right\rangle\right)_{\text {IR }}$ decreases as $t^{-2}$ independently on the value of the power $p$.

\section{Final comments}

In this paper we have studied the evolution of the fluctuations in a symmetric $\phi_{\mathrm{c}}$-exponential potential which provides a power-law expansion using both the gauge-invariant field $\Phi$ and the Sasaki-Mukhanov field. This latter field takes into account simultaneously the inflaton and metric fluctuations. The results obtained from the evolution of $\left(\left\langle\Phi^{2}\right\rangle\right)$ and $\left(\left\langle S^{2}\right\rangle\right)$ are different in both treatments. The reason can be explained from the fact that the field $S=\phi+\frac{\dot{\phi}_{\mathrm{c}}}{H} \Phi$ is not gauge invariant and hence does not describe correctly the fluctuations for $\phi$ and $\Phi$. The fluctuations are well described by the field $\Phi$ which is gauge invariant and predicts a scale invariant power spectrum on the IR sector for $p \rightarrow 2$. Note that we have not considered back-reaction effects which are related to second-order metric tensor fluctuations. This topic was considered by Abramo and Nambu, who investigated a renormalizationgroup method for an inflationary universe $[15,16]$. A different approach to describe the metric fluctuations was considered more recently by Lyth and Wands [17] (see also [18]), who suggested that a curvature perturbation could be generated by a light scalar field named the curvaton.

Acknowledgements. MB acknowledges CONICET, AGENCIA and Universidad Nacional de Mar del Plata for financial support. 


\section{References}

1. A. Guth, Phys. Rev. D 23, 347 (1981)

2. V.F. Mukhanov, H.A. Feldman, R.H. Brandenberger, Phys. Rep 215, 203 (1992)

3. A.A. Starobinsky, in Current Topics in Field Theory, Quantum Gravity, and Strings, edited by H.J. de Vega, N. Sánchez, Lecture Notes in Physics 226 (Springer, New York 1986)

4. Y. Nambu, M. Sasaki, Phys. Lett. B 219 (1989)

5. I. Yi, E.T. Vishniac, Phys. Rev. D 47, 5280 (1993)

6. S. Habib, M. Mijic, UBC report 1991 (unpublished)

7. S. Habib, Phys. Rev. D 46, 2408 (1992)

8. M. Mijic, Phys. Rev. D 49, 6434 (1994)

9. E.J. Copeland, E.W. Kolb, A.R. Liddle, J.E. Lidsay, Phys.
Rev. D 48, 2529 (1993)

10. M. Bellini, H. Casini, R. Montemayor, P. Sisterna, Phys. Rev. D 54, 7172 (1996)

11. S. Matarrese, M.A. Musso, A. Riotto, Influence of superhorizon scales on cosmological observables generated during inflation, preprint: hep-th/0311059

12. M. Sasaki, Prog. Theor. Phys. 76, 1036 (1986); V. Mukhanov, Sov. Phys. JETP, 67, 1297 (1988)

13. A.H. Jaffe et al., Phys. Rev. Lett. 86, 3475 (2001)

14. T.S. Bunch, P.C.W. Davis, Proc. Roy. Soc. A 360, 117 (1978)

15. L.R. Abramo, Phys. Rev. D 60, 064004 (1999)

16. Y. Nambu, Phys. Rev. D 63, 044013 (2001)

17. D.H. Lyth, D. Wands, Phys. Lett. B 524, 5 (2001)

18. T. Moroi, T. Takahashi, Phys. Lett. B 522, 215 (2001) 
Copyright of European Physical Journal C -- Particles \& Fields is the property of Kluwer Academic Publishing / Academic and its content may not be copied or emailed to multiple sites or posted to a listserv without the copyright holder's express written permission. However, users may print, download, or email articles for individual use. 